\title{
POSSIBLE INITIAL EVIDENCE OF EXTRAGALACTIC COSMIC-RAY PROTONS AND

\author{
THE AGE OF EXTRAGALACTIC COSMIC-RAY SOURCES
}

\author{
F. W. STECKER
}

Theoretical Studies Branch, NASA Goddard Space Flight Center, Greenbelt, Md., U.S.A.

\begin{abstract}
We have compared the recent cosmic background $\gamma$-ray observations with spectra predicted by various possible cosmic interactions. We find that the observed isotropic $\gamma$-rays with energies $>1 \mathrm{MeV}$ can best be explained as being due to the decay of $\pi^{\circ}$-mesons produced in extragalactic cosmic-ray collisions. This interpretation indicates that extragalactic cosmic-ray sources were more active (or prevalent) in the past and started to form at a redshift of $\sim 100$ corresponding to $10^{7}-10^{8}$ years after the 'big-bang'.

For a present extragalactic gas density of $10^{-7}-10^{-5} \mathrm{~cm}^{-3}$, the present extragalactic cosmic-ray flux is inferred to be $10^{-5}-10^{-3}$ the galactic value.
\end{abstract}

Recent theoretical studies by the author [1-4] have indicated the importance of observing isotropic cosmic- $\gamma$-radiation in the $1-100 \mathrm{MeV}$ energy region. These predictions of isotropic $\gamma$-ray spectra from metagalactic inelastic strong interactions [1, 3, 4], matter-antimatter annihilation [2], and bremsstrahlung [4], along with studies of metagalactic Compton $\gamma$-rays [5] and bremsstrahlung $\gamma$-rays below $1 \mathrm{MeV}$ energy [6] have indicated the following qualitative points:

(1) Bremsstrahlung and Compton processes may be possible alternative explanations of the observed isotropic $\mathrm{X}$-ray spectrum below $1 \mathrm{MeV}$. The Compton process, however, requires constant regeneration of cosmic-ray electrons [7].

(2) Inelastic proton-proton interactions may account for the observed isotropic $\gamma$-ray flux of Clark et al., [8], if the observed flux is considered to be real, rather than an upper limit. Extrapolations of predicted bremsstrahlung $\left(\sim E_{\gamma}^{-3.6}\right)$ and Compton $\left(\sim E_{\gamma}^{-2.3}\right)$ photon spectra, normalized to fit the X-ray observations, would only be compatible with the measurement of Clark et al. if that measurement is taken as an upper limit due to a spurious signal.

(3) When the predicted $\gamma$-ray spectra were normalized to fit the observations below $1 \mathrm{MeV}$ and above $100 \mathrm{MeV}$ (Clark et al.), it became apparent that a determination of the dominant process, or combination of processes which produce the observed $\mathrm{X}$ and $\gamma$-rays, would only be made possible by a determination of the $\gamma$-ray spectrum between 1 and $100 \mathrm{MeV}$.

The recent observations of Vette et al. [9], have now provided us with measurements of background $\gamma$-rays up to $6 \mathrm{MeV}$. These data, along with some of those of Metzger et al. [10], are shown in the accompanying figure.* The differential intensity at $100 \mathrm{MeV}$ is found from the integral measurement of Clark et al. by assuming that above $100 \mathrm{MeV}$ the spectrum can be approximated by a power law with an index of

* We have also included an upper limit set by a balloon flight of the Rochester group and updated by a recent recalibration (G. Share, private communication). 

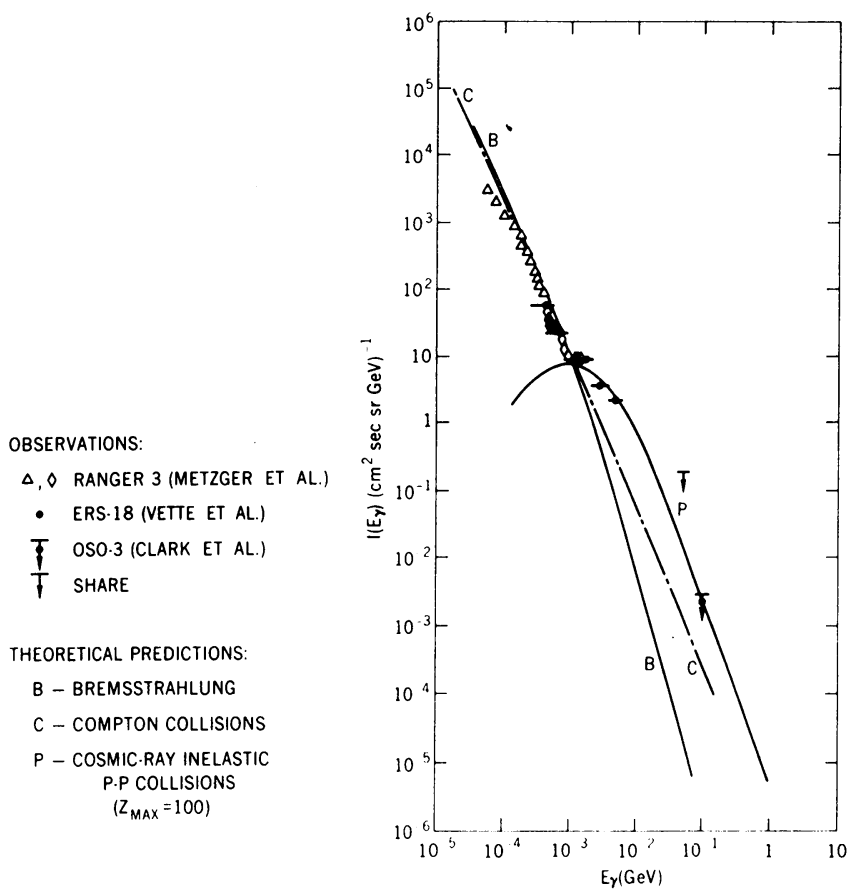

Fig. 1. Extragalactic high energy photon spectra.

$\sim 3$ as shown for the theoretical $\mathrm{p}-\mathrm{p}$ spectrum. Also shown in the accompanying figure, are predicted $\gamma$-ray spectra due to the various possible metagalactic interactions. These spectra have been discussed in detail in References [1-4] and such detailed discussion will not be repeated here.

The new data of Vette et al., are consistent with the power law trend below $1 \mathrm{MeV}$ as indicated by the Ranger 3 measurements and other observations [11]. However, they indicate a marked departure from the power law above $1 \mathrm{MeV}$. For example, the $6 \mathrm{MeV}$ point is an order of magnitude higher than what would be expected on the basis of a power law extrapolation of the X-ray data. These data, taken with the data of Clark et al., being interpreted as a real flux, fit the shape of the theoretical $\gamma$-ray spectrum from $p-p$ interactions integrated to a maximum redshift of $\sim 100$ for a burst or evolving sources model where cosmic-ray production was higher in the past. $[1,4]$. They do not seem consistent with the other theoretical spectra for energies above $1 \mathrm{MeV}$.

These suggestive results make it even more imperative to obtain other $\gamma$-ray observations in the 1-100 MeV region in order to confirm the data of Vette et al., and to extend the measurements to higher energies. However, on the basis of these first results we present the following interpretation.

Comparison of the predicted spectra with the $\gamma$-ray observations indicates that extragalactic $\gamma$-radiation may be due to the decay of neutral pi-mesons produced in inelastic collisions of metagalactic cosmic-ray protons and gas. The peak in the spec- 
trum, which normally occurs at $\sim 70 \mathrm{MeV}$, is redshifted down to $\sim 1 \mathrm{MeV}$ energy. This effect is due to the increased collision rate at larger redshifts when our expanding universe was in a more compact state as well as increased cosmic-ray production at large redshifts. A cosmic-ray production rate which is constant over all reshifts will not account for the new observations [3].

Either a burst model or evolving sources model for the time-dependence of cosmicray production in the past will fit the predicted spectrum; the position of the peak depends primarily on the maximum redshift at which $\gamma$-rays are produced [3]. However, the assumption of various time-dependence models for cosmic-ray production leads to different requirements for the present metagalactic flux needed to produce the observed $\gamma$-rays $[1,4]$. The maximum redshift needed to produce the observations is $\sim 100$, which corresponds to an epoch when the age of the universe was $10^{7}-10^{8}$ years and the temperature of the universal radiation field was $\sim 270 \mathrm{~K}$. This may correspond to the epoch when objects of galactic mass were beginning to form from the metagalactic medium [12]. There is mounting evidence that radio sources were more active (or prevalent) at earlier epochs [13], and it is plausible to speculate that in these sources, where electrons are accelerated to cosmic-ray energies, protons may also be accelerated to these energies. Whereas the electrons have short lifetimes at these redshifts due to Compton interactions with the universal radiation field $[7,14]$ possibly restricting their radio emission stage to redshifts of $\sim 10$ or less, the protons do not undergo significant depletion from Compton interactions. If we consider present extragalactic gas densities of $10^{-5}$ to $10^{-7} \mathrm{~cm}^{-3}$, and assume increased cosmic-ray production in the past, we find that the present intergalactic cosmic-ray flux need only be $\sim 10^{-3}$ $-10^{-5}$ of the galactic value in order to account for the observed $\gamma$-ray intensity. Such a flux has been strongly advocated by Ginzburg and Syrovatskii [15].

\section{References}

[1] Stecker, F. W.: 1968, Nature 220, 675; Corrections: Nature 222, 1157 (1969).

[2] Stecker, F. W.: 1969, Nature 221, 425; Corrections: Nature 222, 1157 (1969).

[3] Stecker, F. W.: 1969, Astrophys. J. 157, 507.

[4] Stecker, F. W. and Silk, J.: 1969, Nature 221, 1229.

[5] Felten, J. E. and Morrison, P.: 1966, Astrophys. J. 146, 686.

Fazio, G. G., Stecker, F. W., and Wright, J. P.: 1966, Astrophys. J. 144, 611.

Gould, R. J.: 1965, Phys. Rev. Letters 15, 511.

Hoyle, F.: 1965, Monthly Notices Roy. Astron. Soc. 120, 338

[6] Silk, J. and McCray, R.: 1969, Astrophys. Letters 3, 59.

[7] Brecher, K. and Morrison, P.: 1969, Astrophys. J. Letters 150, L61.

[8] Clark, G. W., Garmire, G. P., and Kraushaar, W. L.: 1968, Astrophys. J. Letters 153, L203.

[9] Vette, J. I., Gruber, D., Matteson, J. L., and Peterson, L. E.: 1970, this volume, p. 335.

[10] Metzger, A. E., Anderson, E. C., van Dilla, M. A., and Arnold, J. R.: 1964, Nature 204, 766.

[11] See, for example, References in Gould, R. J.: 1967, Am. J. Phys. 35, 376.

[12] Weymann, R.: 1967, Astrophys. J. 147, 887.

[13] Longair, M. S.: 1966, Monthly Notices Roy. Astron. Soc. 133, 421.

Rowan-Robinson, M.: 1968, Monthly Notices Roy. Astron. Soc. 138, 445.

Schmidt, M.: 1968, Astrophys. J. 151, 393.

[14] Bergamini, R., Londrillo, P., and Setti, G.: 1967, Nuovo Cimento 52B, 495.

[15] See discussion and references in Ginzburg, V. L.: 1968, Astrophys. Space Sci. 1, 125. 